\title{
One Ultimate Journey? AKA the Huxley's Method: Perspectives of (Ab)Users of Hallucinogens and Entheogens on Having Planned Pre-Mortem Psychedelic Trip
}

\author{
Ahmed Al-Imam ${ }^{1,2,3,4}$ \& Faris Lami ${ }^{5,6}$ \\ ${ }^{1}$ Department of Anatomy and Cellular Biology, College of Medicine, University of Baghdad, Iraq \\ ${ }^{2}$ CERVO Brain Research Centre, Faculty of Medicine, University of Laval, Canada \\ ${ }^{3}$ The Canadian Association for Neuroscience, Iraq \\ ${ }^{4}$ The Japanese Association of Anatomists, Iraq \\ ${ }^{5}$ Department of Community and Family Medicine, College of Medicine, University of Baghdad, Iraq \\ ${ }^{6}$ Iraq Field Epidemiology Training Program (FETP) Residents Adviser, Iraq \\ Correspondence: Ahmed Al-Imam, Department of Anatomy and Cellular Biology, College of Medicine, \\ University of Baghdad, Bab Al-Mo'adham, 10053, Baghdad, Iraq. E-mail: tesla1452@gmail.com, \\ ahmed.al-imam.1@ulaval.ca
}

Received: Dec. 25, 2019

doi: 10.5539/mas.v13n3p13
Accepted: Jan. 11, 2019

Online Published: February 14, 2019

URL: https://doi.org/10.5539/mas.v13n3p13

\section{Abstract \\ Background}

The surface web is a rich source of extensive data on populations of users and misusers of psychoactive substances including substances known as hallucinogens and entheogens. The internet and its social media websites can serve as a database upon which several hypotheses are applicable via thematic analytic and psychoanalytic studies.

\section{Materials and Methods}

This study will deploy the use of an internet snapshot by inspecting, via thematic analysis, the comments of a population of psychedelic (ab)users existing on the Facebook social platform. The snapshot will dare to answer an existing question in connection with the concept of using psychedelics and entheogens during the moments preceding death. Several demographics will be explored including ethnic-national and socio-cultural parameters to test several hypotheses about the tendencies for having an ultimate pre-mortem psychedelic trip towards the ambiguous afterlife.

\section{Results}

Most of the psychedelic users recommended the use of DMT for the final journey. Others have suggested tripping on LSD, Psilocybin and Psilocybin mushrooms, NBOMe compounds, and even opiates. Based on inferential models, it seems that tendencies for the pre-mortem trip are not affected by the status of social relations, ethnicity, nationality, age, or sex. However, it appears to be based on the individualistic build-up. Religious affiliations and other cultural norms represent potential confounding variables. Hence, these must be explored in subsequent studies

\section{Conclusion}

In the future and to keep in pace with the logarithmic growth and arachnoid expansion of the web and its appendages, ambitious studies has to deploy the use of concepts of automation in data science via the exploitation of principles of machine learning and deep thinking. The aim is to achieve statistical inference in real-time and accurate predictions when it comes to running analytics on big data. If successfully applied, the benefits for the public health should be monumental.

Keywords: Substance-Related Disorders, Psychoactive Substances, Hallucinogens, Psychedelics, (N, N-Dimethyltryptamine), DMT, Psychoanalysis, Internet Snapshot 


\section{Introduction}

Psychoactive substances, including novel psychoactive substances (NPS), represent a varied array of either illegal or regulated chemicals. Many of which can lead to dependence, tolerance, in addition to withdrawal syndrome due to specific pharmacodynamic properties (Dargan \& Wood, 2012; Dargan \& Wood, 2013; Al-Imam, 2017a). Hence, they possess addictive effects that fall under the spectrum of substance use and misuse which is of prime importance from a medical and a forensic science perspective (Al-Imam, Simonato, \& Corazza, 2016; Al-Imam, 2017b; Al-Imam, 2017c; Al-Imam et al., 2017; Al-Imam \& AbdulMajeed, 2017a; Al-Khalisy \& Al-Imam, 2017; Catalani et al., 2018). Over the past two decades, the discipline of neurosciences interested in studying the emerging phenomenon of NPS has been growing at an exponential rate, paralleled only by the analogue pace of explosive growth within the discipline of information and communication technologies (ICT) and its appendageal electronic commerce (e-commerce) spectacle (Ginter, Shear, Spahn, Van Wie, \& Weber, 2014; Turban, King, Lee, Liang, \& Turban, 2015; Meek et al., 2016). Psychoactive substances and NPS could be physiologically categorised into neural activity stimulants, depressants, and a miscellaneous category for chemicals that do not fit into either category (Dargan \& Wood, 2012; Dargan \& Wood, 2013; Al-Imam \& AbdulMajeed, 2017a). On the other hand, the chemical classification is more intricate and beyond the scope of this article (Dargan \& Wood, 2012; Dargan \& Wood, 2013; Al-Imam et al., 2017). Some stimulants, though maybe illegal, are considered cognitive enhancers. Others induce an "elevated state of consciousness" and may be considered "spirituality drugs" (Al-Imam, 2017a; Al-Imam, 2017c; Al-Imam \& AbdulMajeed, 2017a). The latter are hallucinogenic substances commonly known as psychedelics and entheogens (Al-Imam, 2017c; Al-Imam \& AbdulMajeed, 2017b).

Entheogens can induce a state of serenity and a non-describable connection with the universe as subjectively mentioned by most psychedelic users (Al-Imam, 2017d; Al-Imam \& AbdulMajeed, 2017a). Perhaps, it can be considered as a "transcended" state of consciousness on the exact opposite of states like extreme frustration, anger, aggression, and hostility, both physically and mentally (Figure 1) (Dargan \& Wood, 2012; Dargan \& Wood, 2013; Al-Imam, 2017d). One may imagine a state that is completely opposing the Incredible Hulk, a fictional character from Marvel Comics (Sanderson, 1996; Bainbridge, 2010). Psychedelia does not only stand for drugs and chemicals but also represent the entire cultural norms and social interactions related to those chemicals (Lewin, 1991; Echard, 2011). This study aims to reach a conclusive inference concerning the propensity of psychedelic (ab)users towards having an intentional psychedelic trip in the moments of pre-mortem. Truly, some renowned individuals attempted the ultimate pre-mortem psychedelic tripping, including Aldous Leonard Huxley who was a prominent novelist and philosopher (Beauchamp, 1990). Huxley, at the age of 69 years, while suffering from an advanced form of laryngeal cancer requested his wife to administer an intramuscular injection of LSD before his death (Dyck, 2015; Sessa, 2015). Hence, intentional tripping before death can be termed "the Huxley's Method". It is possible that other famous individuals and scientists have also experimented with psychedelics including Leonardo Da Vinci, Sigmund Freud, and Thomas Edison (Famousscientists.org, 2018; Shroomery.org, 2018). Our study requires a keen observational or quasi-experimental approach towards the mentality of (ab)users of entheogens via thematic (psycho)analysis (Pérez, Crick, \& Lawrence, 2015; Bell, 2017; Blacker, Kurtz, \& Goodwin, 2017).

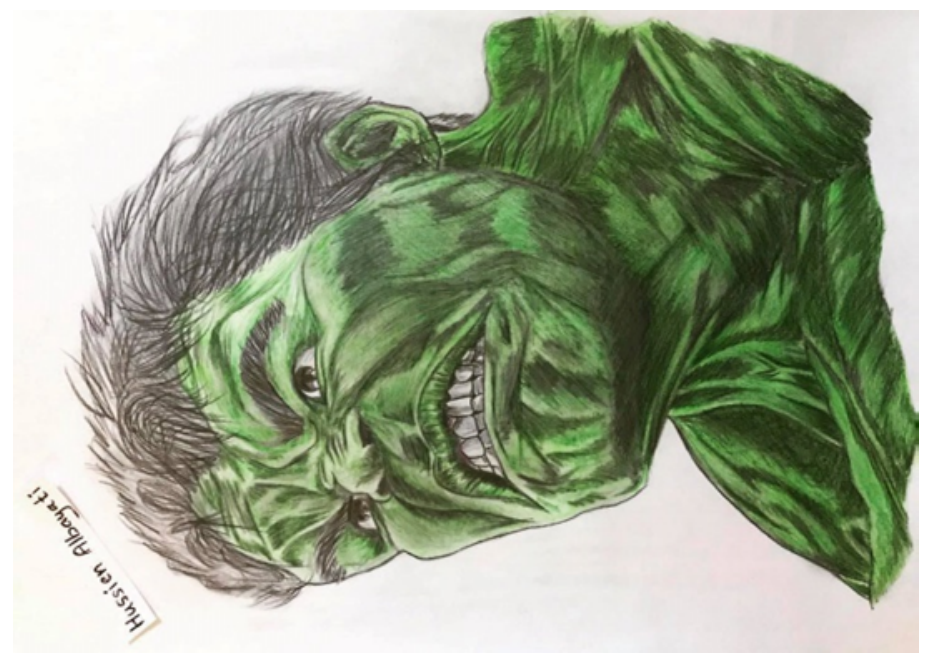

Figure 1. The Incredible Hulk: Fictional Character in Marvel Comics 
$\dagger$ Concept Art by Hussein Al-Bayati, Medical Student at the College of Medicine-University of Baghdad.

\section{Materials and Methods}

\subsection{Ethical Aspects}

This study has been ethically permitted by the Institute Review Board (IRB) of the College of Medicine at the University of Baghdad and under the authority of the IRB meeting number seven which took place on the $20^{\text {th }}$ of December 2016.

\subsection{Population and Inclusion Criteria}

This study is observational and cross-sectional in design which relies on a hybrid approach based on qualitative and quantitative tools. It is based on an internet snapshot, which is a technique of taking a "screenshot" at a given moment of time for data available on a particular website. A series of internet snapshots were taken during October 2017 for a specific thread which was posted on a private group (The Terence McKenna Experience) for psychedelics (ab)users existing on the popular online social media platform known as Facebook (Facebook.com, 2018). The snapshots were taken for the commentaries of psychedelics (ab)users of different backgrounds, age, sex, ethnicity, and nationality. The comments were in correspondence to a question that was already posted by another member of the online community. The existing question was formulated as "Dosing on psychedelics before death, thoughts?".

\subsection{Data Collection}

Members of the group actively participated in replying to the query of interest. Comments were either supporting tripping (pro-tripping, numerically scored as "2"), against tripping (anti-tripping, numerically scored as "0") or had a neutral attitude (numerically scored as "1") towards tripping via the specific use of DMT (N, N-Dimethyltryptamine) or other entheogens prior to death. The themes of commentaries were evaluated using thematic (psycho)analysis (Pérez et al., 2015; Bell, 2017; Blacker et al., 2017). Hence, this study aims to extrapolate evidence via the dissection of the personal accounts and opinions of psychedelic (ab)users. The research questions to be answered via this analysis include: Are the psychedelics users favouring tripping using psychedelics (including DMT and LSD) before death? Are there any precautions and recommendations? Should certain factors interfere? These factors may involve the timing and place of the psychedelic trip, the availability of a companion known as a "trip sitter", type of substance to be used, the route of substance administration, association with suicidal ideations and intentions, and the avoidance of specific venues and objects while tripping.

\subsection{Data Entry and Analysis}

There will be tabulation of variables including the demographic parameters of interest, the use of multimedia in commentaries, raw attitude-tendency towards the concept of pre-mortem tripping (anti-tripping, undecided, or pro-tripping), density of speech expressed as the number of words per a comment (WPC), and the number of themes-recommendations implied within a comment for each (ab)user. Further, a quantitive score termed the attitude index will be calculated based on speech density, the use of multimedia, the number of suggested themes of recommendations, and the attitude. This score (index) was later used for statistical inference via testing the effect of sex, age, ethnicities, nationalities versus tendencies towards having an ultimate pre-mortem trip. The level-of-evidence for this study is estimated to be of level-4 in compliance with the categorisation scheme of the level-of-evidence imposed by the Oxford Center for Evidence-Based Medicine (OCEBM Levels of Evidence Working Group, 2011). A systematic review of the literature was carried out via medical and paramedical databases of literature including NCBI-PubMed, the Cochrane Library, Embase, EBSCO, ResearchGate, and Academia. The grey literature databases were also consulted in parallel with relevant data from social media and electronic media (e-media), media networks, blogs, and online drug communities (fora) including Erowid, Bluelight, Reddit, and Drugs-Forum (Drugs-forum.com, 2018; Erowid.com, 2018; Officialbenzofury.net, 2018; Reddit.com, 2018). The reviewed literature of interest was subsequently scrutinised for credibility and reliability via the implementation of the appropriate tools of critical appraisal (Glynn, 2006). Statistical analyses were carried out via Microsoft Excel 2016 and the Statistical Package for Social Sciences (SPSS v.24, 64-bits Operating System). The implemented statistical tests included parametric and non-parametric tests at an alpha value $(\alpha)$ of 0.05 as the cut-off margin for statistical significance.

\section{Results}

Commentaries of all psychedelic users ( $\mathrm{n}=119)$ were considered, while duplicate comments were excluded. The mean values for the screened parameters were 36.49 years (age), 13.69 words (speech density), 0.06 (incorporation of multimedia in comments), 1.46 (attitude), 1.03 (number of suggestions per user), and 28.72 
(attitude index). Concerning the range of these parameters, it was 18-67 years (age), 0-90 (words per comment), 0-1 (either incorporated the use of multimedia or not), 0-2 (anti-tripping, undecided, or pro-tripping), 0-5 (number of suggestions and themes per comment), and 0-88.61 (attitude index). Further, statistical outliers (Figure 2) were detected for age (67 years old, male, Caucasian, married, from the United States, 21 WPC, pro-tripping attitude, and attitude index of 35.83). Concerning the attitude index, a single outlier was observed (24 years old, male, Caucasian, not in-relation, from the US, 67 WPC, pro-tripping attitude, and attitude index of 88.61). Concerning the speech density, there were eight (8) statistical outliers (33-90 WPC). Those outliers were related strictly to Caucasian individuals $(8 / 8)$, one of which was a female $(1 / 8)$, and all were from the United States (US) while two individuals were from the United Kingdom (UK) and Finland. Half of which were married (4/8), and none of them had an anti-tripping attitude (0/8).

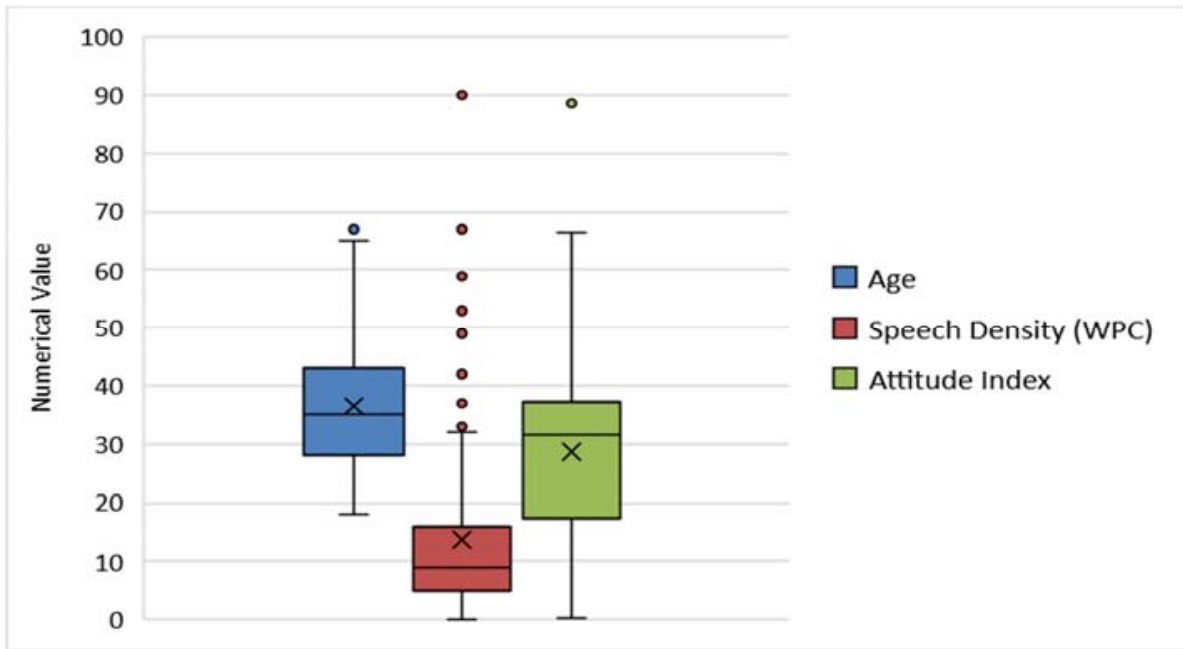

Figure 2. Boxplot Presentation for Age, Density of Speech, and the Attitude Index.

In relation to age groups, class intervals reveal that the vast majority were $18-28$ years $(n=30,25.21 \%), 28-38$ years of age $(n=43,36.14 \%), 38-48$ years $(n=32,26.89 \%)$ summing up to $88.24 \%$. Hence, the majority of individuals are in between the $2^{\text {nd }}$ and the $5^{\text {th }}$ decades of life. Males and females contributed to $89 \%$ ( $\left.n=106\right)$ and $11 \%(n=13)$ respectively. Individuals were distributed internationally (Figure 3) into twenty countries, most of them were from the US $(n=81,68.07 \%)$, UK $(n=11,9.24 \%)$, Canada $(n=4,3.36 \%)$, and Australia $(n=3,2.52 \%)$ summing up for $83.19 \%$. Other nationalities included citizens from Romania, Norway, Netherlands, India, Denmark, France, Sweden, Alaska, Puerto Rico, Ireland, Spain, Brazil, Finland, New Zealand, Guatemala, and Cyprus. Accordingly, most of the psychedelic users were from the developed world, and none of them was from the Middle East. Ethnic groups strictly included Caucasians $(\mathrm{n}=101,84.87 \%)$, Latins $(\mathrm{n}=14,11.77 \%)$, and Asians $(n=4,3.36 \%)$. The majority were single $(n=77,64.71 \%)$.

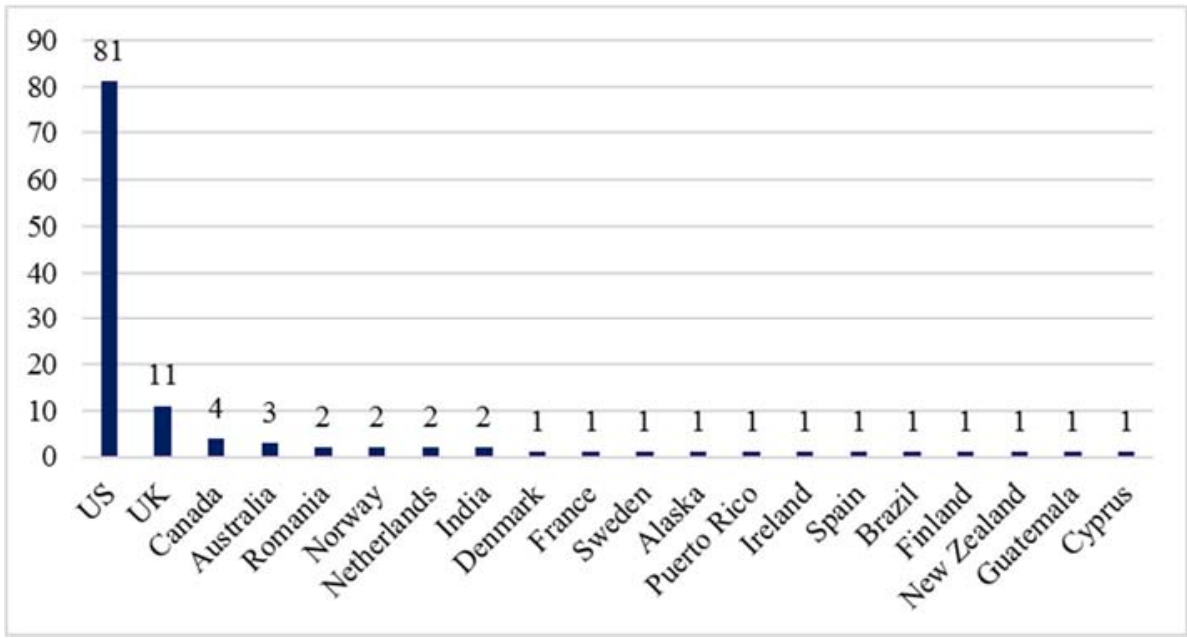

Figure 3. Descriptives: International Contribution 
Based on sex (Figure 4), the mean value for attitude is 1.69 (females) and 1.43 (males), while averaging 30.38 (women) and 28.51 (men) for the attitude index which may indicate (hypothesis-1) that females could possess a more pro-tripping tendency than males. Based on ethnic grouping (Figure 5), the summative attitude index was distributed as 85\% (Caucasian), 13\% (Latin), and 2\% (Asian) which could be either due to the higher contribution of individuals from Caucasian ethnicity or due to a stronger pro-tripping attitude among Caucasians. Further, in relation to ethnicity, the mean value for attitude index is 30.6 (Latins), 28.8 (Caucasian), and 20 (Asians). Accordingly, Latin individuals may have a higher pro-tripping attitude (hypothesis-2). To summarise, two hypotheses emerged, the $1^{\text {st }}$ should test the significant difference in opinions based on sex, while the $2^{\text {nd }}$ should verify the existence of a considerable difference based on ethnicity via examining the dominant contributors (Caucasian versus Latin ethnic groups). A $3^{\text {rd }}$ and $4^{\text {th }}$ hypothesis will also consider the statistical inference based on social-marital relations (single individuals versus those in-relation), and nationality by comparing the top two contributors (the US versus the UK). A $5^{\text {th }}$ hypothesis will test the raw (initial) attitude scores (anti-tripping, neutral, and pro-tripping).

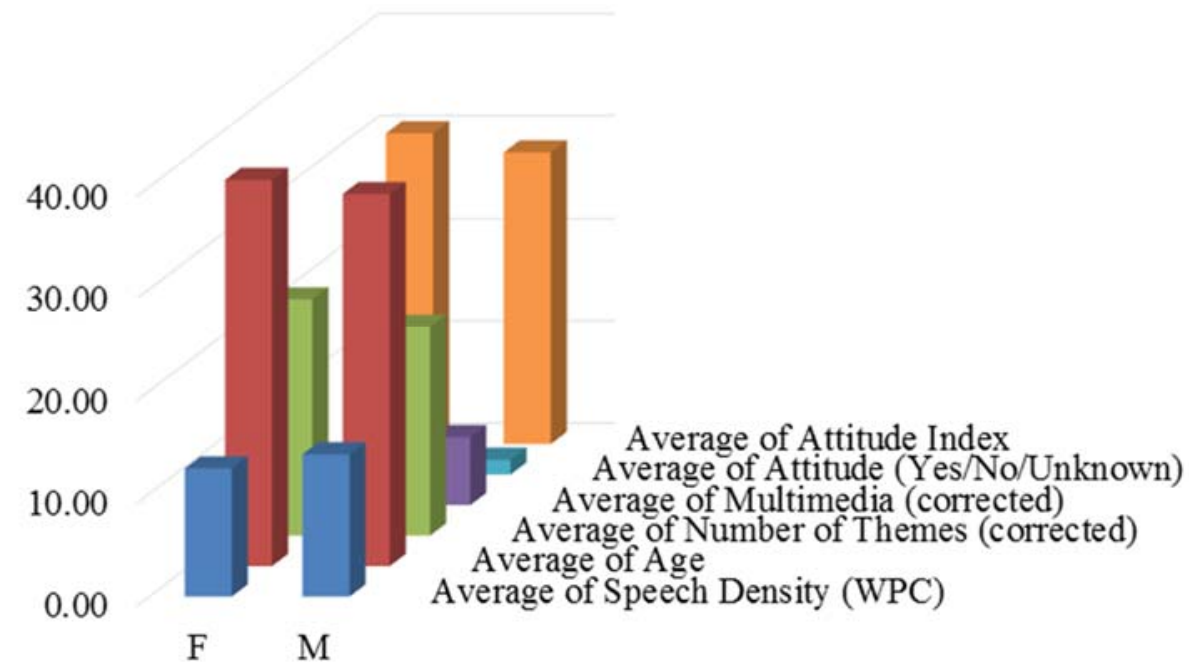

Figure 4. Descriptive Parameters Based on Sex

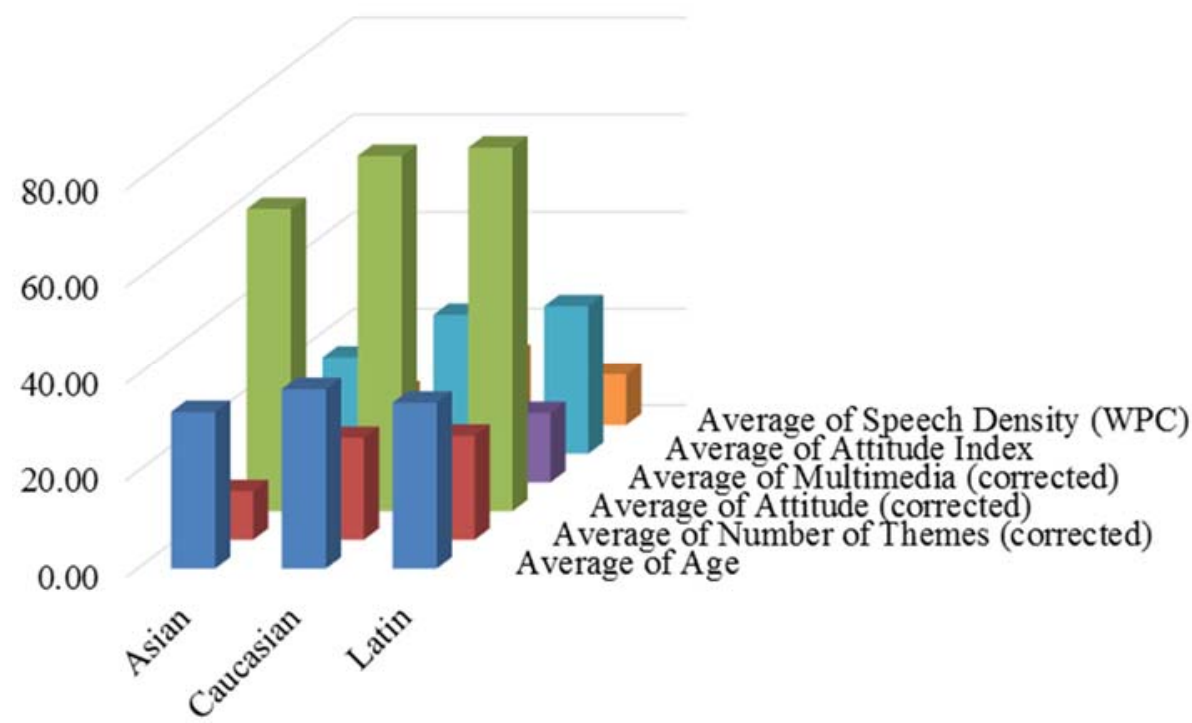

Figure 5. Descriptive Parameters Based on the Ethnicity

Descriptive parameters, related to speech density and the attitude index (Figure 6), visualise a potential correlation of the attitude index versus speech density. It seems that psychedelic users with anti-tripping attitude generated more extended comments (mean value of WPC $=20.44$ ), while those of pro-tripping and undetermined attitude commented less (13.75 and 9). Hence, individuals with anti-tripping attitude tend to elaborate on their 
ideas about the concept. On the other hand, individuals with undetermined attitude were very brief in their commentaries giving a short explanation of why they took a neutral stance. Accordingly, an ultimate $\left(6^{\text {th }}\right)$ hypothesis will be tested, via regression models, to correlate the attitude index with its subcomponents (speech density, raw attitude scoring, the incorporation of multimedia in commentaries, and the number of themes-suggestions per a comment).

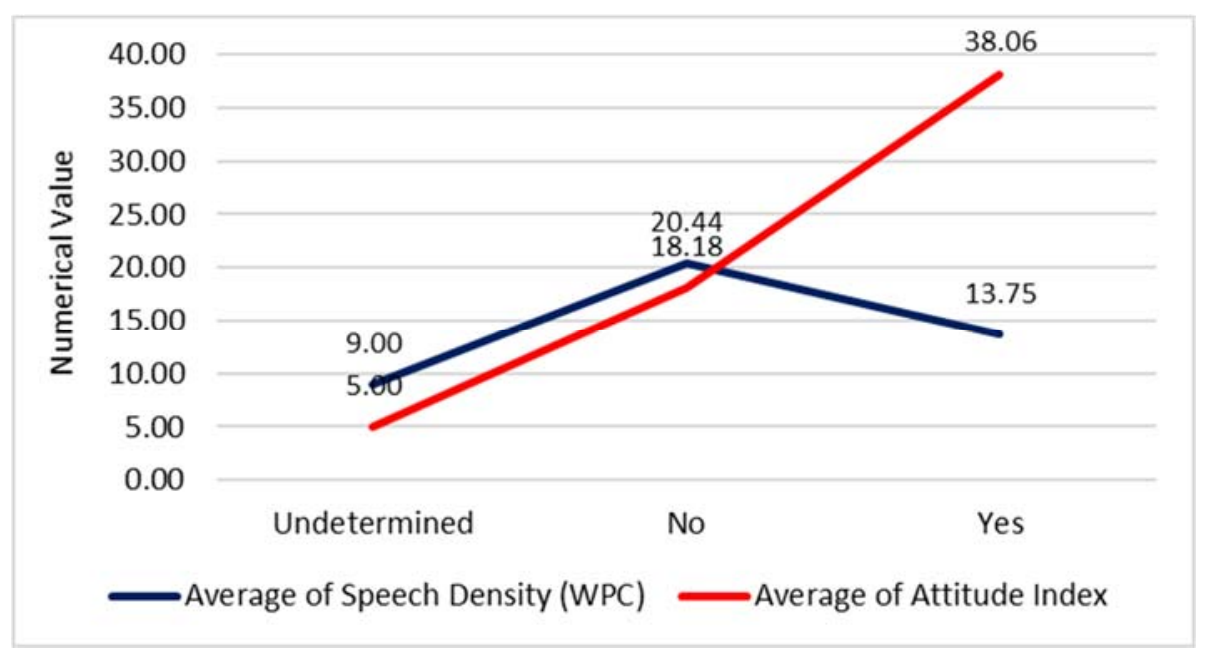

Figure 6. The Correlation of the Attitude Index versus the Density of Speech

Concerning the $1^{\text {st }}$ hypothesis, it is inferred that sex (males versus females) has no significant effect on age, speech density, raw attitude scoring, number of themes and suggestions, and the attitude index. However, it has a significant differential effect on the incorporation of multimedia in commentaries. The use of multimedia was more frequent in males than in females ( $p$-value $=0.008)$ which could be attributed to the sexual dimorphism in the neural structure and physiology of the brain as men are considered as visual thinkers while women are more into abstract thinking. Concerning the $2^{\text {nd }}$ hypothesis, ethnic grouping (Caucasians versus Latins) has not displayed any significant difference in connection with age, speech density, the incorporation of multimedia, number of suggestions, raw attitude scoring, and the attitude index. Hence, ethnicity was not a determinant parameter towards tendencies for pre-mortem psychedelic tripping. Concerning the $3^{\text {rd }}$ hypothesis, it is inferred that the social-marital status (single versus in-relation) does not affect speech density, the use of multimedia, number of themes, raw attitude scoring, and attitude index. However, there was a significant difference in age between those who are single versus those who are in-relation (34.66 versus $39.79 \mathrm{p}=0.021$ ). Married individuals were significantly older by roughly five years (5.13 years). The age was not a determinant parameter towards deciding to weather trip before death or not.

Concerning the $4^{\text {th }}$ hypothesis (US versus UK), nationality has no significant determinant effect neither on the attitude index or its subcomponents. In other words, citizenship does not affect the decision-making process about pre-mortem psychedelic tripping. Concerning the $5^{\text {th }}$ hypothesis (Table 1), it seems that the initial (raw) scoring of attitudes can significantly affect the attitude index as well as the speech density and the number of themes per a comment. Initial scoring (screening) of the attitude is always a significant determinant of the ultimate attitude index. Accordingly, individuals labelled as pro-tripping have a significantly higher attitude index than individuals who were tagged as undecided $(\mathrm{p}<0.001)$. Similarly, individuals tagged as undecided have a considerably higher attitude index than individuals who were labelled as anti-tripping $(\mathrm{p}<0.001)$. Concerning the $6^{\text {th }}$ hypothesis, the highest significant correlation of the attitude index exists with raw attitude scoring $\left(\mathrm{R}^{2}\right.$ score $=0.6831)$ and the number of suggestions $\left(\mathrm{R}^{2}=0.6812\right)$, while the lowest exists with the inclusion of multimedia $\left(\mathrm{R}^{2}=0.1739\right)$ and speech density $\left(\mathrm{R}^{2}=0.1475\right)$. To summarize, the attitude index is mainly affected by the raw attitude scoring and the number of themes (suggestions) per comment. Males tend to embed the use of multimedia as they opine on the topic but without affecting their tendencies towards pre-mortem tripping. On the other hand, ethnicity, nationality, and the social-marital status never had a significant effect on those tendencies either. The most critical determinant of the attitude index was found to be the raw attitude scoring itself as confirmed via inferential statistics. 
Table 1. The Significant Effect of Raw Attitude Scoring on the Attitude Index and Collateral Parameters.

\begin{tabular}{lccc}
\hline \multicolumn{3}{c}{$p$-value } \\
\hline & Neutral vs. Anti-tripping & Neutral vs. Pro-tripping & Anti-tripping vs. Pro-tripping \\
Age & 0.628 & 0.536 & 0.990 \\
Speech Density & $\mathbf{0 . 0 0 9}$ & $\mathbf{0 . 0 3 7}$ & 0.114 \\
Multimedia & 0.162 & 0.756 & $\mathbf{0 . 0 2 4}$ \\
Number of Themes & 0.328 & $<\mathbf{0 . 0 0 1}$ & $<\mathbf{0 . 0 0 1}$ \\
Attitude Index & $<\mathbf{0 . 0 0 1}$ & $<\mathbf{0 . 0 0 1}$ & $<\mathbf{0 . 0 0 1}$ \\
\hline
\end{tabular}

$\dagger$ Significant $p$-values are typed in bold fonts.

\section{Discussion}

The concept of this study is genuine and has never been explored before in the existing body of literature. Future studies should attempt achieving a homogenous and balanced sampling while assuring biases are minimized. Sampling should also be conducted via stratification and clustering while exploring more than one population of psychedelic users existing on social e-media, blogs, and online drug fora. Attempts should also be exploited in exploring the invisible web (deep web) and the darknet to test whether there are some discrepancies from the surface web. Further, trends databases, including Google Trends, can be explored in connection with the topic of pre-mortem psychedelic tripping to assess the digital epidemiology and geography of people interested in the subject. It is also essential to inspect the concepts of cerebral dominance (lateralisation of brain functions), manifested as patterns of handedness in the population, that may interfere with the decision-making process (Al-Hadithi, Al-Imam, Irfan, Khalaf, \& Al-Khafaji, 2016; Al-Hadithi \& Al-Imam, 2017; Al-Imam, 2017e; Al-Imam, 2018; Al-Imam, Ali, \& Saad, 2018). Additionally, online and offline surveys and interviews should be deployed to reduce the reliance on web snapshot technique that is prone to errors and biases. Besides, solitary and group pressure (psychology concept of conformity) must be applied to infer stress-induced variations on the decision-making process (De Dreu \& Kret, 2016; Romano \& Balliet, 2017).

Cultural norms and religious affiliations (atheism, agnosticism, monotheism, polytheism, etc.) of psychedelic (ab)users should be carefully investigated as these may interfere with sensitive topics related to death and the afterlife. Miscellaneous studies can also explore populations of psychedelia enthusiasts and users from the developing world including the Middle East and the Far East, as well as the developed world. Studies should also be dedicated to exploring the visual patterns of thinking and spoken-written expressions of language in correspondence to the sex of psychedelic (ab)users and correlating it with their decision-making concerning having an intentional final psychedelic journey. Refinement and advancement of the applied statistical methods for hypothesis testing to incorporate the application of multivariate analyses and logistic regression models. More advanced and anticipated studies may integrate automation in data science and concepts of machine learning for real-time analytics and prospective predictive inference (Al-Imam, 2019; Al-Imam, Khalid, Al-Hadithi, \& Kaouche, 2019). Those algorithms could be of value in communities who are sensitive to discuss similar topics in an aim to reduce the biases while conducting a simple snapshot of the population of interest especially those from developing countries as well as conservative and religious societies.

\section{Conclusion}

For psychedelic (ab)users, there are different tendencies towards having an ultimate trip using psychedelics and entheogens. Individuals could have a pro-tripping, an anti-tripping, or even be undecided about the concept of having a pre-mortem psychedelic trip. Those tendencies can be investigated via thematic exploration of linguistic expressions made by those individuals. It was found that sex, ethnicity, nationality, and social relationships do not interfere with the decision-making process of the studied concept. This study can be considered as a primordial blueprint for future studies to be implemented for real-time and predictive analytics of a specific population of users existing on the web. Applying concepts of automated data science, including machine learning, will serve thematic analytic studies great leverage while dwarfing the requirement of human and financial resources to a minimum. The majority of psychedelics users recommended the use of DMT, LSD, psilocybin, opiates, and some variants of NBOMe compounds. The study does have limitations including the sole reliance on data retrieved from the surface web, and the implementation of Facebook as a representative of social e-media existing on the internet. Further, only one private group was explored on Facebook. Besides, the 
study was based on an internet snapshot and not a classical survey or an interview. Additionally, all comments were explored by-distance via an online system, and the sample was not homogenous especially concerning ethnic grouping and citizenship. Only written texts (commentaries) were analysed which could introduce some information biases during data collection and interpretation.

\section{Acknowledgements}

The author would like to acknowledge the efforts of Hussein Al-Bayati, a medical student and a promising junior doctor, for the graphic concept art of the Incredible Hulk (Figure 1). The author would also like to acknowledge, with gratitude, an online community existing on Facebook by the name the Terence McKenna Experience.

\section{Conflicts of Interest}

The authors have no competing interests.

\section{Source of Funding}

This study is entirely self-funded.

\section{References}

Al-Hadithi, N., \& Al-Imam, A. (2017). (Dis) Inclination of Iraqi Medical Students Towards Creative Thinking: A Quasi-Experimental Study. Global Journal of Health Science, 9(11), 186.

Al-Hadithi, N., Al-Imam, A., Irfan, M., Khalaf, M., \& Al-Khafaji, S. (2016). The relation between cerebral dominance and visual analytic skills in Iraqi medical students, a cross sectional analysis. Asian Journal of Medical Sciences, 7(6), 47-52.

Al-Imam, A. (2017a). Monitoring and Analysis of Novel Psychoactive Substances in Trends Databases, Surface Web and the Deep Web, with Special Interest and Geo-Mapping of the Middle East. info:eu-repo/semantics/masterThesis [dissertation on the Internet]. United Kingdom: University of Hertfordshire; 2017. https://doi.org/10.13140/RG.2.2.27636.24961

Al-Imam, A. (2017b). Retrospective Analyses of High-risk NPS: Integrative Analyses of PubMed, Drug Fora, and the Surface Web. Global Journal of Health Science, 9(11), 40.

Al-Imam, A. (2017c). Could Hallucinogens Induce Permanent Pupillary Changes in (Ab) users? A Case Report from New Zealand. Case Reports in Neurological Medicine, 2017.

Al-Imam, A. (2017d). Let's Go Out For A Day Trip? Perspectives of Psychedelics (Ab) Users on the Safety of Acid (LSD) Tripping in Public Places. Global Journal of Health Science, 9(11), 156.

Al-Imam, A. (2017e). The relation between cerebral dominance and visual analytic skills in Iraqi Medical students, a cross sectional analysis. Journal of the Anatomical Society of India, 66(1), 42-43.

Al-Imam, A. (2018). Lateralization of Brain Functions: A Randomized Single-Blinded Observational Study. Iranian Journal of Psychiatry and Behavioral Sciences, 12(1).

Al-Imam, A. (2019). A Gateway Towards Machine Learning: Predictive Analytics and Neural Networks in IBM-SPSS (SPSS v.24). Retrieved January 3, 2019 from https://www.researchgate.net/publication/329029699_A_Gateway_Towards_Machine_Learning_Predictive _Analytics_and_Neural_Networks_in_IBM-SPSS_SPSS_v24. https://doi.org/10.13140/RG.2.2.20885.99041/3

Al-Imam, A., \& AbdulMajeed, B. A. (2017a). Novel Psychoactive Substances: Systematic Review and Evidence-Based Analysis of Literature. Global Journal of Health Science, 9(11), 1.

Al-Imam, A., \& AbdulMajeed, B. A. (2017b). Do (Ab) Users of Psychedelics Have Tendencies to Abuse High Authority? An Internet Snapshot Study. Global Journal of Health Science, 9(11), 148.

Al-Imam, A., Ali, H., \& Saad, A. (2018). Face Recognition Abilities in Iraqi Medical Students: An Inferential, Cross Sectional Analysis. Iranian Journal of Psychiatry and Behavioral Sciences, 12(1).

Al-Imam, A., Khalid, U., Al-Hadithi, N., \& Kaouche, D. (2019). Real-time Inferential Analytics Based on Online Databases of Trends: A Breakthrough Within the Discipline of Digital Epidemiology of Dentistry and Oral-Maxillofacial Surgery. Modern Applied Science, 13(2), 81-94.

Al-Imam, A., Santacroce, R., Roman-Urrestarazu, A., Chilcott, R., Bersani, G., Martinotti, G., \& Corazza, O. (2017). Captagon: use and trade in the Middle East. Human Psychopharmacology: Clinical and Experimental, 32(3), e2548. 
Al-Imam, A., Simonato, A. P., \& Corazza, O. (2016). Haloperidol, an old antipsychotic with potential use by NPS users in Iraq. Research and Advances in Psychiatry, 3(3), 81-84.

Al-Khalisy, M., \& Al-Imam, A. (2017). Commercial Acetic Acid: A Potential Regenerative Agent for Chronic Cutaneous Wounds? A Quasi-Experimental Study. Global Journal of Health Science, 9(11), 178.

Bainbridge, J. (2010). "I Am New York"-Spider-Man, New York City and the Marvel Universe. Comics and the City, New York: Continuum, 163-79.

Beauchamp, G. (1990). Island: Aldous Huxley's Psychedelic Utopia. Utopian Studies, 1(1), 59-72.

Bell, C. R. (2017). Psychotherapeutic Subjectivities: A Thematic Analysis of Experiences of Change in Psychoanalysis/Psychodynamic Psychotherapy and Cognitive-Behavioral Therapy (Doctoral dissertation, University of West Georgia).

Blacker, R., Kurtz, A., \& Goodwin, A. (2017). An in-depth observational study of an acute psychiatric ward: combining the psychodynamic observational method with thematic analysis to develop understanding of ward culture. Psychoanalytic Psychotherapy, 31(1), 4-20.

Catalani, V., Prilutskaya, M., Al-Imam, A., Marrinan, S., Elgharably, Y., Zloh, M., ... \& Corazza, O. (2018). Octodrine: new questions and challenges in sport supplements. Brain Sciences, 8(2), 34.

Dargan, P. I., \& Wood, D. M. (2012). Recreational drug use in the Asia Pacific region: improvement in our understanding of the problem through the UNODC programmes. Journal of Medical Toxicology, $8(3)$, 295-299.

Dargan, P., \& Wood, D. (Eds.). (2013). Novel psychoactive substances: classification, pharmacology and toxicology. Academic Press.

De Dreu, C. K., \& Kret, M. E. (2016). Oxytocin conditions intergroup relations through upregulated in-group empathy, cooperation, conformity, and defense. Biological Psychiatry, 79(3), 165-173. Drugs-forum.com. Retrieved 11 April 2018 from https://drugs-forum.com/index.php

Dyck, E. (2015). LSD: a new treatment emerging from the past. Canadian Medical Association Journal, 187(14), 1079-1080.

Echard, W. (2011). Psychedelia, musical semiotics, and environmental unconscious. Green Letters, 15(1), 61-75.

Erowid. Erowid.com. Retrieved 11 April 2018 from https://www.erowid.org/

Facebook.com (2018). The Terence McKenna Experience. Retrieved 11 April 2018 from https://www.facebook.com/Psychedelia.Heaven/?sw_fnr_id=3989766998\&fnr_t $=0$

Famousscientists.org (2018). 14 Famous Scientists and Inventors who Experimented with Drugs. Retrieved 11 April 2018

from https://www.famousscientists.org/14-famous-scientists-inventors-who-experimented-with-drugs/

Ginter, K. L., Shear, V. H., Spahn, F. J., Van Wie, D. M., \& Weber, R. P. (2014). U.S. Patent No. 8,751,793. Washington, DC: U.S. Patent and Trademark Office.

Glynn, L. (2006). A critical appraisal tool for library and information research. Library Hi Tech, 24(3), 387-399.

Greenhalgh, T., Howick, J., \& Maskrey, N. (2014). Evidence based medicine: a movement in crisis? British Medical Journal, 348, g3725.

Lewin, R. (1991). Stone Age psychedelia: Was the cave art of Stone Age people the fruit of hallucinations induced by shamanistic rituals. New Scientist, 1772, 30-43.

Meek, C. A., Horvitz, E. J., Goodman, J. T., Flake, G. W., Hurst-Hiller, O., Gupta, A., ... \& Griffin, T. J. (2016). U.S. Patent No. 9,396,269. Washington, DC: U.S. Patent and Trademark Office.

OCEBM Levels of Evidence Working Group. (2011). The Oxford 2011 Levels of Evidence. Oxford Centre for Evidence-Based Medicine. Retrieved from http://www. cebm. net/index. aspx?o= 5653

Officialbenzofury.net (2018). Retrieved 11 April 2018 from http://officialbenzofury.net/

Pérez, A., Crick, P., \& Lawrence, S. (2015). Delving into the 'emotional storms': A thematic analysis of psychoanalysts' initial consultation reports. The International Journal of Psychoanalysis, 96(3), 659-680.

Reddit.com (2018). Retrieved 11 April 2018 from https://www.reddit.com/

Romano, A., \& Balliet, D. (2017). Reciprocity Outperforms Conformity to Promote Cooperation. Psychological 
Science, 28(10), 1490-1502.

Sanderson, P. (1996). Marvel Universe (p. 209). Harry N. Abrams.

Sessa, B. (2015). Continuing history of psychedelics in medical practices: The renaissance of psychedelic medical research. The Psychedelic Policy Quagmire: Health, Law, Freedom, and Society, 61-92.

Shroomery.org. (2018). Da Vinci \& Michelangelo Used Psychedelics? Retrieved 11 April 2018 from https://www.shroomery.org/forums/showflat.php/Number/18857370

Turban, E., King, D., Lee, J. K., Liang, T. P., \& Turban, D. C. (2015). Overview of electronic commerce. In Electronic Commerce (pp. 3-49). Springer, Cham.

\section{Copyrights}

Copyright for this article is retained by the author(s), with first publication rights granted to the journal.

This is an open-access article distributed under the terms and conditions of the Creative Commons Attribution license (http://creativecommons.org/licenses/by/4.0/). 